\title{
"The evaluation of derivatives of double barrier options of the Bessel processes by methods of spectral analysis"
}

\begin{tabular}{|c|c|}
\hline AUTHORS & $\begin{array}{l}\text { Ivan Burtnyak (D https://orcid.org/0000-0002-9440-1467 } \\
\text { Anna Malytska (D https://orcid.org/0000-0002-5811-9288 }\end{array}$ \\
\hline ARTICLE INFO & $\begin{array}{l}\text { Ivan Burtnyak and Anna Malytska (2017). The evaluation of derivatives of double } \\
\text { barrier options of the Bessel processes by methods of spectral analysis. } \\
\text { Investment Management and Financial Innovations, 14(3), 126-134. } \\
\text { doi:10.21511/imfi.14(3).2017.12 }\end{array}$ \\
\hline DOI & http://dx.doi.org/10.21511/imfi.14(3).2017.12 \\
\hline RELEASED ON & Wednesday, 11 October 2017 \\
\hline RECEIVED ON & Monday, 10 July 2017 \\
\hline ACCEPTED ON & Monday, 21 August 2017 \\
\hline LICENSE & $\begin{array}{l}\text { The EY-No } \\
\text { This work is licensed under a Creative Commons Attribution-NonCommercial } 4.0 \\
\text { International License }\end{array}$ \\
\hline JOURNAL & "Investment Management and Financial Innovations" \\
\hline ISSN PRINT & $1810-4967$ \\
\hline ISSN ONLINE & $1812-9358$ \\
\hline PUBLISHER & LLC "Consulting Publishing Company "Business Perspectives" \\
\hline FOUNDER & LLC "Consulting Publishing Company "Business Perspectives" \\
\hline
\end{tabular}

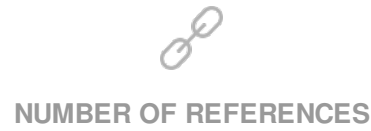

18

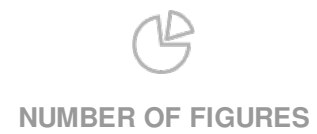

1
NUMBER OF TABLES

0

(C) The author(s) 2022. This publication is an open access article. 


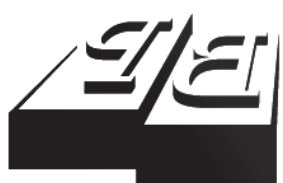

BUSINESS PERSPECTIVES

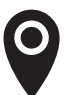

LLC "CPC "Business Perspectives" Hryhorii Skovoroda lane, 10, Sumy, 40022, Ukraine

www.businessperspectives.org

Received on: $10^{\text {th }}$ of July, 2017 Accepted on: $21^{\text {st }}$ of August, 2017

(c) Ivan Burtnyak, Anna Malytska, 2017

Ivan Burtnyak, Ph.D. in Economics, Associate Professor, Department of Economic Cybernetics, Vasyl Stefanyk Precarpathian National University, Ukraine.

Anna Malytska, Ph.D. in Physics and Mathematics, Associate Professor, Department of Mathematical and Functional Analysis, Vasyl Stefanyk Precarpathian National University, Ukraine.

\section{(ㄷ)(1) $(8$}

This is an Open Access article, distributed under the terms of the Creative Commons Attribution-NonCommercial 4.0 International license, which permits re-use, distribution, and reproduction, provided the materials aren't used for commercial purposes and the original work is properly cited.

\section{THE EVALUATION OF DERIVATIVES OF DOUBLE BARRIER OPTIONS OF THE BESSEL PROCESSES BY METHODS OF SPECTRAL ANALYSIS}

\begin{abstract}
The paper deals with the spectral methods to calculate the value of the double barrier option generated by the Bessel diffusion process. This technique enables us to calculate the option price in the form of a Fourier-Bessel series with the corresponding ratio The autors propose a simple method to estimate options using the Green's expansion function for boundary value problem for a singular parabolic equation. Thus, the accuracy of the estimation coincides with the accuracy of the convergence of the FourierBessel series.

In this paper, the authors use the spectral theory to calculate the price of derivatives of financial assets considering that the processes are described by Markov and can be considered in Hilbert spaces. In this work, the authors use the diffusion process to find derivatives prices by introducing them through the Bessel functions of first kind. They also examine the Sturm-Liouville problem where the boundary conditions utilize the Bessel functions and their derivatives. All assumptions lead to analytical formulae that are consistent with the empirical evidence and, when implemented in practice, reflect adequately the passage of processes on stock markets.

The authors also focus on the financial flows generated by Bessel diffusion processes which are presented in the system of Bessel functions of the first order under the condition that the linear combination of the flow and its spatial derivative are taken into account. Such a presentation enables us to calculate the market value of a share portfolio, provides the measurement of internal volatility in the market at any given time, and allows us to investigate the dynamics of the stock market.
\end{abstract}

The splitting of Green's function in the system of Bessel functions is presented by an analytical formula which is convenient for calculating the price level of options.
Keywords

JEL Classification spectral theory, barrier option, financial flows, Bessel diffusion process, Bessel functions, Green's function, singular parabolic operator, infinitesimal operator

\section{INTRODUCTION}

The study of the nature and functioning of the fundamentals of options was performed by many scientists, among which should be noted: Merton (1973), Cox et al. (1976), Lorig (2014). General theory developed different approaches to the evaluation of derivatives and one double barrier option depending on assumption stochastic processes they describe, the greatest theoretical achievement in the field of derivatives was to create an option pricing model, known worldwide as a Black-Scholes model (Black \& Scholes, 1973). To estimate the value of options, a binomial model was created, which was constructed by Sharpe (1985), which was later generalized by Cox et al. (1985). Recently, a number of new models of pricing options have appeared, 
but there is not always an analytical formula for the image of the solution, so we assume that the processes are Markov processes.

Problems in research of barrier options were discussed by the following scientists: Hull and White (1987), Davydov and Linetsky (2003), Gorovoi and Linetsky (2004), Mendoza-Arriaga et al. (2010).

Bessel processes are most often used in the theory of mass service, in particular in the theory of queues. Stochastic Bessel processes are of different type with respect to the problems on their own values and their own responsibilities when spectrum of Bessel is continuous or has a finite number of values and continuous part is presenting density through the functions of Whittaker's and Laguerre polynomials considered in many papers and most detailed by Linetsky (2004), where the boundary value problem on the interval $x \in(0, \infty)$ with boundary conditions imposed on the first derivative of the density function for $x \rightarrow \infty$ is considered.

Bessel processes play an important role in financial mathematics, because by nature closely related to the geometric Brownian motion model and processes CIR (Cox-Ingersoll-Ross), they allow a clear representation of the density transition including bond prices and options and facilitate statistical evaluation of process parameters (Cox et al., 1985). Under certain characteristics of the diffusion process, Bessel operator never becomes zero. Several works of Yor (1984) were devoted to these cases. In this paper we consider the cases where derivatives of Bessel processes flow may become zero.

Under these conditions is explained, the excess rate of growth of stock portfolio is determined and the excess rate of growth of the market portfolio provide which a measurement of the internal market volatility at any given time (Linetsky, 2007).

The paper by Coffman et al. (1998) describes the application of Bessel processes in the financial markets and the relation with the integral of the geometric Brownian motion model. Going-Jaeschke and Yor (2003), review was conducted on the pricing of Asian options using Bessel processes.

The diffusion with the Bessel operator was investigated by Davydov and Linetsky (2003), but under other boundary conditions, and in orthogonal systems of functions.

\section{RESEARCH METHODOLOGY}

In this paper, we consider one-dimensional diffusion with Bessel process with a drift, which is zero (there is a number of processes of this type where the drift is not equal to zero, but the research can be reduced to processes with zero drift). Such processes are used in solving economic problems in finding short-term interest rates, credit spreads and stochastic volatility of derivatives (GoingJaeschke et al., 2003).

The problem of this type is considered for the first time. For such problems, there is an unsolved issue when Bessel diffusion has nonlocal volatility dependent on various factors, but such an issue is only partially solved for ordinary diffusion process- es generated by the Brownian motion. Therefore, new approaches and analysis are already required for solving problems of this type.

The approach developed by us can be applied to the research for the pricing of barrier options generated by Bessel processes. To do this, we must consider the financial flows generated by Bessel diffusion processes by expanding the distribution density (the Green's function of the corresponding problem) into the Fourier-Bessel series. Such a development gives ample opportunities for the application of this theory, because the Bessel functions and their derivatives are tabulated and well-studied. We can calculate the size of a market share portfolio and determine the level of internal volatility in the market at any given time, and examine the dynamics of the stock market. 
The spectral method is applied to derivative financial instruments, pricing through the presentation of the price of the derivative asset $u(t, x)$ neutral to the risk of waiting for some function from the future value of the main process $X$. Process $X$ may represent many economic processes. For example, the size of stocks, the price of the index, a reliable short percentage, etc.

Let us consider the process for which the operator $L$ has the form

$$
L=\partial_{x x}^{2}+x^{-1} \partial_{x}-x^{-2} p^{2},
$$

where $p$ is constant, which is called index, $x>0$.

We note that $L$ is a singular parabolic operator, infinitesimal, self-conjugate, and a number of operators are reduced to it, where $\sigma^{2}=2 x^{2}$, and $L$ is Bessel operator.

The diffusion with Bessel operator was investigated in works of Linetsky (2004), Davydov et al. (2003), but under other boundary conditions, and in systems of orthogonal functions.

To study $L$ on eigenvalues and eigenfunctions, under certain boundary conditions, we consider the Bessel equation.

$$
x^{2} \cdot v^{\prime \prime}+x \cdot v^{\prime}+\left(x^{2}-p^{2}\right) \cdot v=0 .
$$

The solution of equation (2), except for the partial values of $p$, is not expressed through elementary functions (in finite form), these non-elementary functions are called Bessel functions. They are widely used in economics, technics and physics. Since the Euler-Bessel equation is linear, its general solution can be written as follows:

$$
v=C_{1} \cdot v_{1}+C_{2} \cdot v_{2},
$$

where $v_{1}, v_{2}$ are two arbitrary linearly independent partial solutions of Euler-Bessel equation, and $C_{1}, C_{2}$ are arbitrary constant.

In case of $p \geq 0$, we make the substitution $v=x^{p} \cdot w$, and get an equation for function $w$

$$
w^{\prime \prime}+\frac{2 p+1}{x} \cdot w^{\prime}+w=0
$$

The solution of the obtained equation is a formal power series, which is absolutely convergent for all $x \in(-\infty ; \infty)$, that is

$v=x^{p} w=\frac{\left(\frac{x}{2}\right)^{p}}{\Gamma(p+1)}+$

$+\sum_{m=1}^{\infty} \frac{(-1)^{m}\left(\frac{x}{2}\right)^{p+2 m}}{12 \ldots m(p+1) \ldots(p+m) \Gamma(p+1)}$,

where $\Gamma$ is a Gamma function. By transforming (5) on the basis of the Gamma function properties, we obtain the Bessel function of the first kind, of the order $p$.

$$
J_{p}(x)=\sum_{m=0}^{\infty} \frac{(-1)^{m}\left(\frac{x}{2}\right)^{p+2 m}}{\tilde{\mathrm{A}}(m+1) \cdot \tilde{\mathrm{A}}(p+m+1)} .
$$

Remark. Since $p^{2}$ is included in equation (2), the change of $p^{2}$ to $p$ does not affect the solution of the equation, therefore, there is a solution for arbitrary $p$.

If $p$ is not an integer, then the Bessel functions cannot be linearly dependent and the general integral of equation (2), is as follows:

$$
J=C_{1} \cdot J_{p}(x)+C_{2} \cdot J_{-p}(x),
$$

for integral $p$, we find another partial solution

$$
Y_{p}(x)=\frac{J_{p}(x) \cos p \pi+J_{-p}(x)}{\sin p \pi},
$$

which is the Bessel function of the second kind, which is indefinite for $x=0$ using the L'Hopital rule, we find the boundary for $x \rightarrow 0$ and by this number we define the function at zero

$$
\begin{aligned}
& Y_{0}=\frac{2}{\pi} \cdot J_{0}(x)\left(\ln \frac{x}{2}+C\right)- \\
& -\frac{2}{\pi} \sum_{m=1}^{\infty}(-1)^{m}\left(\frac{x}{2}\right)^{2 m} \cdot\left(1+\frac{1}{2}+\frac{1}{3}+\ldots+\frac{1}{m}\right) .
\end{aligned}
$$


For any $p$, there are formulae

$$
\begin{aligned}
& \frac{d}{d x} \cdot\left(x^{p} \cdot J_{p}(x)\right)=x^{p} \cdot J_{p-1}(x), \\
& \frac{d}{d x} \cdot\left(x^{-p} J_{p}(x)\right)=-x^{-p} \cdot J_{p+1}(x) .
\end{aligned}
$$

The Bessel functions $J_{p}(\lambda x), J_{p}(\mu x)$ where $\lambda$ and $\mu$ are the roots of the equation $J_{p}(x)=0$, are orthogonal on the interval $[0,1]$ weighing $h x$ that is

$$
\int_{0}^{1} x J_{p}(\lambda x) J_{p}(\mu x) d x=0,
$$

$\lambda \neq \mu$ and for $\lambda=\mu$, two cases are possible

$$
\int_{0}^{1} x J_{p}^{2}(\lambda x) d x=\left\{\begin{array}{l}
\frac{1}{2} J_{p}^{\prime 2}(\lambda), J_{p}(\lambda)=0 \\
\frac{1}{2}\left(1-\frac{p^{2}}{\lambda^{2}}\right) J_{p}^{2}(\lambda), J_{p}^{\prime}(\lambda)=0 .
\end{array}\right.
$$

For all $\alpha, \beta \geq 0, \alpha+\beta>0$, there exists a denumerable set of positive roots $\alpha v_{k}^{\prime}(\mu)+\beta \mu v_{k}(\mu)=0$, which frontier point is on infinity.

Let $v(x)$ be a solution (2), then the function $v(\lambda x)$ will also be a solution of the equation of this sort

$$
x^{2} v^{\prime \prime}+x v^{\prime}+\left(\lambda^{2} x^{2}-p^{2}\right) v=0
$$

Equation (13) is the Bessel equation with the parameter $\lambda$.

Any solution of equation (2), which is the Bessel function, has an infinite set of positive roots, which are close to the roots of the function $\sin (x+\omega)$, the form $k_{n}=n \pi-\omega, \omega=$ const, $n$ - integer (similarly for negative roots, as they are symmetrically relative to the origin of coordinates), if $k_{n} \neq 0$, then they are simple roots and form a denumerable set.

Bessel functions are alternating sign-rows, so the evaluation can be made using the Leibniz lemma, by which we can determine the accuracy of the approximation.
To find eigenfunctions and eigenvalues let us, consider such a boundary value problem

$$
\begin{aligned}
& x^{2} v_{k}^{\prime}+x v_{k}^{\prime}+\left(\lambda_{k}^{2} x^{2}-p^{2}\right) v_{k}=0, \\
& x^{2} v_{k}^{\prime \prime}+x v_{k}^{\prime}+\left(\lambda_{k}^{2} x^{2}-p^{2}\right) v_{k}=0, \\
& \left|v_{k}\right|_{x=0}<+\infty
\end{aligned}
$$

$\alpha v_{k}^{\prime}\left(x_{0}\right)+\beta v_{k}\left(x_{0}\right)=0$.

So, we consider the Sturm-Liouville problem. This problem has a single solution. We impose problem situation (6), because $x=0$ is a special point of equation (5) and the operator $L . x_{0}$ is a regular point of equation (5). Those values $\lambda_{k}$ for which boundary value problems (5)-(7) have a nontrivial solution $v_{k}$ are called eigenvalues, and $v_{k}$ are eigenfunctions of the problem. It is known that under problem situations (6), the operator $L$ has a numerable number of eigenfunctions, they are simple and nonnegative (Vladimirov, 1981). Multiplying $L$ on $x^{2}$ does not change neither eigenvalues nor eigenfunctions or their numbers.

Let us consider this problem

$$
\left\{\begin{array}{l}
x^{2} v_{k}^{\prime \prime}+x v_{k}^{\prime}+\left(\lambda_{k}^{2} x^{2}-p^{2}\right) v_{k}=0 \\
\left|v_{k}\right|_{x=0}<+\infty \\
v_{k}\left(x_{0}\right)=0
\end{array}\right.
$$

From (6) it follows that $p \geq 0$, let us consider the case $p>0$. It is easy to verify that $\lambda=0$ is not an eigenvalue of the problem (8) (N. N. Lebedev, 1972).

Since(2)hasitsintegral $v=C_{1} \cdot J_{p}(x)+C_{2} \cdot Y_{p}(x)$ then on the basis of properties of the Bessel function, problem (8) has the solution

$$
v=C_{1} J_{p}(\lambda x)+C_{2} \cdot Y_{p}(\lambda x)
$$

Keeping in mind the boundary conditions, we have $C_{2}=0$ and $C_{1} J_{p}\left(\lambda_{k} x_{0}\right)=0$ that is $J_{p}\left(\lambda_{k} x_{0}\right)=0$, hence, ${ }_{\grave{l}} \quad \lambda_{k} x_{0}=\mu_{k}$, where $\quad J_{p}\left(\mu_{k}\right)=0$, $\lambda_{k}=\frac{\grave{l}_{k}}{x_{0}}, \quad 0<\mu_{1}<\mu_{2}<\ldots<\mu_{k}<\ldots$, so 
$v_{k}(x)=J_{p}\left(\frac{\mu_{k}}{x_{0}} x\right), k=1,2, \ldots$

Considering the case where $p=0$ we obtain such problem

$$
\left\{\begin{array}{l}
\left(x v^{\prime}\right)^{\prime}+\lambda^{2} x v=0 \\
|v|_{x=0}<+\infty \\
v\left(x_{0}\right)=0
\end{array}\right.
$$

For $\lambda=0$, we have $\left(x v^{\prime}\right)^{\prime}=0$, hence, it follows that $x v^{\prime}=C_{1}$ or $\frac{d v}{d x}=\frac{C_{1}}{x}$ so the general integral has the form $v=C_{1} \ln x+C_{2}$. According to a boundedness condition, we have that $v_{0}=C_{2}$, from the boundary conditions we conclude that $v=0$, hence $\ddot{e}=0$ is not an eigenvalue.

Let $\lambda>0$, then the general solution has the form

$$
v=C_{1} J_{0}(\lambda x)+C_{2} Y_{0}(\lambda x) .
$$

Since the Bessel function of the second kind $Y_{0}(\lambda x)$ is infinite at zero, then $C_{2}=0$, so $v(x)=C_{1} J_{0}(\lambda x)$, meeting the boundary conditions, we observe $J_{0}\left(\lambda x_{0}\right)=0$. We receive the roots of this equation $\mu_{k}, 0<\mu_{1}<\mu_{2}<\ldots<\mu_{k}<\ldots$

$$
\lambda_{k}=\frac{\mu_{k}}{x_{0}}, v_{k}(x)=J_{0}\left(\frac{\mu_{k}}{x_{0}} x\right), k=1,2, \ldots
$$

Note that the functions $1, J_{p}\left(\frac{\mu_{k}}{x_{0}} x\right), k=1,2, \ldots$ are orthogonal with each other on $\left[0, x_{0}\right]$ with weight $x$.

Let us consider the Bessel process described

$$
\begin{aligned}
& \frac{\partial v(t, x)}{\partial t}=\frac{\partial^{2} v(t, x)}{\partial x^{2}}+x^{-1} \frac{\partial v(t, x)}{\partial x}- \\
& -p^{2} x^{-2} v(t, x), 0<x<x_{0},
\end{aligned}
$$

and the boundary condition

$v(0, x)=K\left(e^{x}-1\right)^{+}, v\left(t, x_{0}\right)=0$,

where $K$ is strike, the process is homogeneous, that is why $v(t, x)=\varphi(t) \cdot v(x)$.

From the Sturm-Liouville theory it follows that

$v(t, x)=\sum_{n=1}^{\infty} c_{n p} e^{-\frac{\mu_{n}^{2}}{x_{0}^{2}} t} \cdot J_{p}\left(\frac{\mu_{n}}{x_{0}} x\right), p \geq 0$,

where $\mu_{n}$ - positive roots of the equation $J_{p}\left(\mu_{n} x_{0}\right)=0, c_{n p}$ - coefficients of the expansion in the Fourier-Bessel series of functions $K\left(e^{x}-1\right)$.

The financial flows are as follows:

$u(t, x)=\sum_{n=1}^{\infty} K c_{n p} \cdot e^{-\left(\frac{\mu_{n}}{\ln K}\right)^{2}(T-t)} \cdot J_{p}\left(\mu_{n} \ln \frac{x}{K}\right)$,

in case, if the process is completed at the time $T$, when $X_{T}=K$, then

$u(t, x)=\sum_{n=0}^{\infty} K c_{n} e^{-\left(\frac{\mu_{n}}{\ln \frac{R}{L}}\right)^{2}(T-t)} \cdot J_{p}\left(\frac{\mu_{n}\left(K-\ln \frac{x}{L}\right)}{\ln \frac{R}{L}}\right)$,

where $L<x<R, L, R$ are barriers, $K$ is a strike, and $c_{n p}$ are calculated as follows:

$c_{n p}=2 K \frac{\int_{0}^{1} t\left(e^{K t}-1\right) \cdot J_{p}\left(\mu_{n} t\right) d t}{J_{p+1}^{2}\left(\mu_{n}\right)}$.

We calculated the decomposition of the financial flow in the system of Bessel functions $J_{p}$ of the first kind, but the distribution of flows is set by Green's function of the corresponding problem. Therefore, it is convenient for calculating to expand the Green's function in the Bessel system. The process we consider corresponds with heterogeneous boundary value problem 
$\frac{\partial u}{\partial t}=\frac{\partial^{2} u}{\partial x^{2}}+x^{-1} \frac{\partial u}{\partial x}-$

$-\frac{p^{2} u(t, x)}{x^{2}}+f(t, x), x>0$

where $f(t, x)$ is twice continuously differentiable with respect to $x$ continuously differentiable with respect to $t$, is absolutely integrable with derivative, $(t, x) \in[0,+\infty)$ and may be written as

$f(t, x)=\sum_{n=0}^{\infty} f_{n}(t) \cdot J_{p}\left(\frac{\mu_{n} x}{x_{0}}\right)$,

$0<x<x_{0}<+\infty, 0<t<T$,

$\mu_{n}$ is the equation root $J_{p}\left(\mu_{n}\right)=0$.

The solution of this problem will be sought in the form

$u(t, x)=\sum_{n=0}^{\infty} T_{n}(t) \cdot J_{p}\left(\frac{\mu_{n} x}{x_{0}}\right)$,

substituting in (12) we obtain

$$
\begin{aligned}
& \sum_{n=0}^{\infty} T_{n}^{\prime}(t) J_{p}\left(\frac{\mu_{n} x}{x_{0}}\right)=\sum_{n=0}^{\infty}\left\{\left[\left(J_{p}\left(\frac{\mu_{n} x}{x_{0}}\right)\right)_{x^{2}}^{\prime \prime}\right.\right. \\
& +\frac{\left(J_{p}\left(\frac{\mu_{n} x}{x_{0}}\right)\right)_{x}^{\prime}}{x}-\frac{p^{2} J_{p}\left(\frac{\mu_{n} x}{x_{0}}\right)}{x^{2}}+ \\
& \left.\left.+\lambda_{n}^{2} J_{p}\left(\frac{\mu_{n} x}{x_{0}}\right)\right]-\lambda_{n}^{2} J_{p}\left(\frac{\mu_{n} x}{x_{0}}\right)\right\} T_{n}(t)+ \\
& +\sum_{n=0}^{\infty} f_{n}(t) J_{p}\left(\frac{\mu_{n} x}{x_{0}}\right),
\end{aligned}
$$

therefore

$$
\sum_{n=0}^{\infty}\left[T_{n}^{\prime}(t)+\lambda_{n}^{2} T_{n}(t)-f_{n}(t)\right] J_{p}\left(\frac{\mu_{n} x}{x_{0}}\right) \equiv 0
$$

where
$T_{n}^{\prime}(t)+\lambda_{n}^{2} T_{n}(t)-f_{n}(t)=0, \lambda_{n}=\frac{\mu_{n}}{x_{0}}, n \in N$,

with the initial condition $T_{n}(0)=0$.

The inhomogeneous differential equation of first order is by the method of variation of parameters. Since $T_{n}^{\prime}(t)+\lambda_{n}^{2} T_{n}(t)=0$ has the first integral $T_{n}(t)=C e^{-\lambda_{n}^{2} t}$, solving the inhomogeneous equation by the method of variation of parameters observer $T_{n}(t)=\int_{0} e^{-\lambda_{n}^{2}(t-\beta)} f_{n}(\beta) d \beta$, therefore

$u(t, x)=\sum_{n=0}^{\infty} \int_{0}^{t} e^{-\lambda_{n}^{2}(t-\beta)} f_{n}(\beta) d \beta J_{p}\left(\frac{\mu_{n} x}{x_{0}}\right)$.

Taking into account that

$$
\begin{aligned}
& f_{n}(t)=\int_{0}^{x_{0}} \xi f_{n}(\xi, t) J_{p}\left(\frac{\mu_{n} \xi}{x_{0}}\right) \times \\
& \times d \xi\left(\int_{0}^{x_{0}} x J_{p}^{2}\left(\frac{\mu_{n} x}{x_{0}}\right) d x\right)^{-1},
\end{aligned}
$$

we obtain

$$
\begin{aligned}
& u(t, x)=\sum_{n=0}^{\infty} \int_{0}^{t} e^{-\lambda_{n}^{2}(t-\beta)} \int_{0}^{x_{0}} \xi f(\xi, t) J_{p}\left(\frac{\mu_{n} \xi}{x_{0}}\right) \times \\
& \times d \beta d \xi J_{p}\left(\frac{\mu_{n} x}{x_{0}}\right)\left(\int_{0}^{x_{0}} y J_{p}^{2}\left(\frac{\mu_{n} y}{x_{0}}\right) d y\right)^{-1}= \\
& =\int_{0}^{x_{0}} \int_{0}^{t} \sum_{n=0}^{\infty}\left(y J_{p}^{2}\left(\frac{\mu_{n} y}{x_{0}}\right) d y\right)^{-2} e^{-\lambda_{n}^{2}(t-\beta)} \xi J_{p} \times \\
& \times\left(\frac{\mu_{n} \xi}{x_{0}}\right) J_{p}\left(\frac{\mu_{n} x}{x_{0}}\right) f(\xi, t) d \xi d \beta,
\end{aligned}
$$

that is Green's function $G(t-\beta, x, \xi)$ has the form

$$
\begin{aligned}
& G(t-\beta, x, \xi)=\sum_{n=0}^{\infty} \xi J_{p}\left(\frac{\mu_{n} \xi}{x_{0}}\right) J_{p}\left(\frac{\mu_{n} x}{x_{0}}\right) \times \\
& \times e^{-\frac{\mu_{n}^{2}}{x_{0}}(t-\beta)}\left(\frac{x_{0}^{2}}{2} J_{p+1}^{2}\left(\mu_{n}\right)\right)^{-1},
\end{aligned}
$$




\section{APPLICATION}

\section{OF THE DESCRIBED \\ METHODOLOGY}

Since the problem of evaluation and research of two-dimensional barrier options is reduced to the study and solution of the boundary value problem, (Burtnyak et al., 2013, 2016).

$$
\begin{aligned}
& \frac{\partial u(t, x)}{\partial t}=\frac{\partial^{2} u(t, x)}{\partial x^{2}}+x^{-1} \frac{\partial u(t, x)}{\partial x}- \\
& -\frac{p^{2} u(t, x)}{x^{2}}, x \in[L, H], t \in[0, T],
\end{aligned}
$$

$u(t, L)=0, u(t, H)=0$,

$u(T, x)=\max ( \pm(x(T)-K), 0) \mathbb{I}_{(L<x(t)<H ; t \in[0, T]}$.

$y=\ln x$,

$y \in[A, B], t \in[0, T]$,

$A=\ln L, B=\ln H$,

$$
\begin{aligned}
& u(t, A)=0, u(t, B)=0, \\
& u(0, y)=\psi\left(e^{y(T)}\right)= \\
& =\max ( \pm(x(T)-K), 0) \mathbb{I}_{(L<x(t)<H ; t \in[0, T])} .
\end{aligned}
$$

Taking into account all the arguments for seeking a solution of the classical boundary value problems for a singular parabolic operator $L$, we obtain that

$u(T, x)=\int_{0}^{\ln \frac{H}{L}}\left(e^{\xi} L-K\right) \mathbb{I}_{(L<x(t)<H ; t \in[0, T])} \times$

$\times G(x, \xi) d \xi=\int_{0}^{\ln \frac{H}{L}}\left(e^{\xi} L-K\right) \mathbb{I}_{(L<x(t)<H ; t \in[0, T])}$,

$\frac{\partial u}{\partial t}=\frac{\partial^{2} u}{\partial y^{2}}+y^{-1} \frac{\partial u}{\partial y}-\frac{p^{2} u(t, x)}{y^{2}}$

$y=\ln x$,

$y \in[A, B], t \in[0, T]$,

$2 \sum_{n=0}^{\infty} e^{-\left(\frac{\mu_{n}}{\ln \frac{H}{L}}\right)^{2} t} J_{p}\left(\frac{\mu_{n} \xi}{\ln \frac{H}{L}}\right) J_{p}\left(\frac{\mu_{n} \ln \frac{x}{L}}{\ln \frac{H}{L}}\right) \times$

$A=\ln L, B=\ln H$,

$\times\left(\ln \frac{H}{L}\right)^{-2}\left(J_{p+1}^{2}\left(\mu_{n}\right)\right)^{-1}$,

where $\mathbb{I}_{(L<x(t)<H ; t \in[0, T])}$ is Heaviside step function.

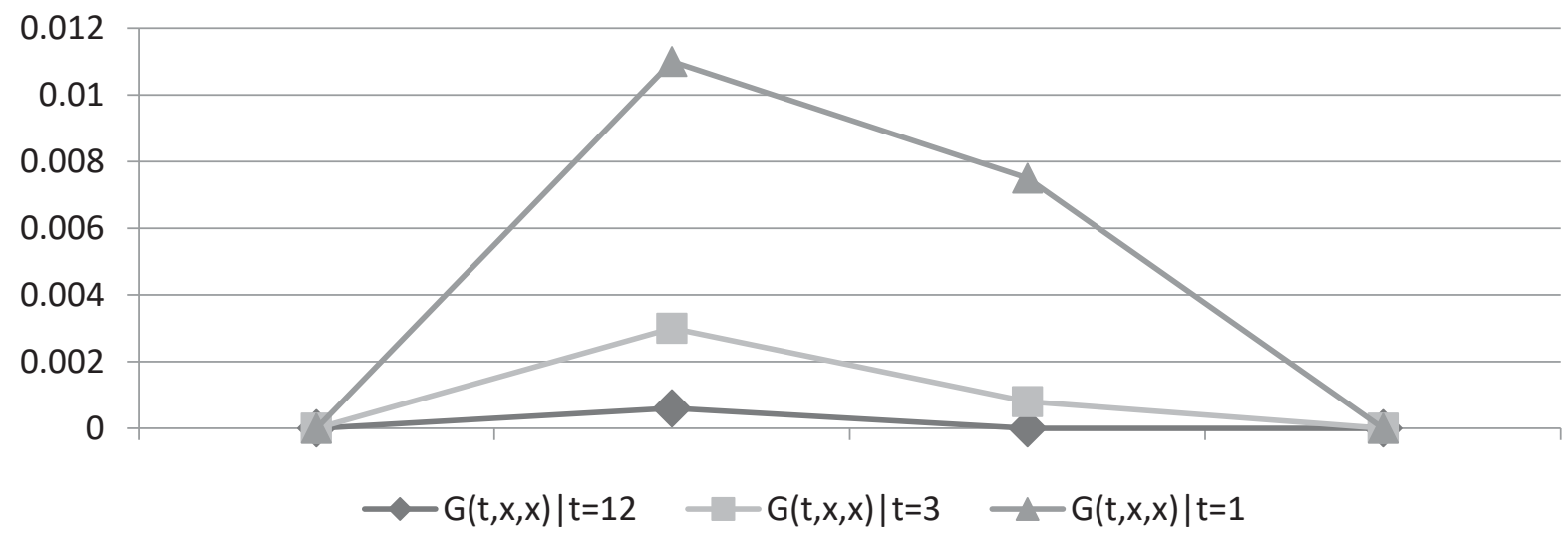

Figure 1. Graph of Green's function as density distribution at $L=90, H=120, \xi=0.5$ 
Remark. Since the roots of Bessel functions of the first order are close to the roots of the function $\sin (x+\omega)$, the form $k_{n}=n \pi-\omega, \omega=$ const, $n$ - integer, then at large $n, \mu_{n}^{2}$ can be changed by $n^{2}$. Hence, it follows that series for Green's function and its first and second derivatives coincide proportionally. Therefore, at approximate calculations, due to rapid convergence, it does not require a large number of series coefficients.

$$
\left.\left.\left.G(t, x, \xi)\right|_{t=1} G(t, x, \xi)\right|_{t=3} G(t, x, \xi)\right|_{t=12}
$$

\section{CONCLUSION}

One-dimensional diffusion is used to describe the primary dynamics of the stock market, and the spectral method serves as a powerful tool for analytical pricing.

The spectral theory is used to find the price of derivatives of financial assets, considering that the pro-

cesses described by Markov and such that they can be considered in Hilbert spaces $L^{2}$, using the SturmLiouville theory. Bessel diffusion processes are used in the study of barrier options. We considered the financial flows generated by Bessel diffusion processes by introducing them in the system of Bessel functions of the first type. Such a presentation enables us to calculate the size of a market share portfolio and provides measurement of internal volatility in the market at any given time, allows us to investigate the dynamics of the stock market.

In this paper, we have constructed the Green's function for the Bessel diffusion process of a two-barrier option, which is expanded in the Bessel functions system. Since the Bessel functions are alternating sign-rows, the calculation of derivative financial instruments prices can be carried out with arbitrarily high accuracy. The Green's function is written analytically and its representation is convenient and does not cause difficulties when calculating derivatives prices.

\section{REFERENCES}

1. Black, F., \& Scholes, M. (1973). The Pricing of Options and Other Corporate Liabilities. Journal of Political Economy. 81(3), 637-654. Retrieved from https://www. cs.princeton.edu/courses/archive/ fall09/cos323/papers/black_scholes73.pdf

2. Carr, P., \& Linetsky, V. (2006). A Jump to Default Extended CEV Model: An Application of Bessel Processes. Finance and Stochastics, 10, 303-330.

3. Coffman, E. G., Puhalskii, A. A., \& Reiman, M. I. (1998). Polling systems in heavy traffic: a Bessel process limit. Math. Operat. Res., 23, 257-304.

4. Cox, J. C., Ross, S., Rubinstein, M. (1976). The Valuation of options for alternative stochastic processes. Journal of Financial economics, 3, 145-166.
5. Cox, J. C., Ingersoll, J. E., Ross, S. A. (1985). A theory of the term structure of interest rates. Econometrica, 53(2), 385-408. Retrieved from http://www.fin.ntu. edu.tw/ tzeng/course/CIR(1985)Eca.pdf

6. Davydov, D., \& Linetsky, V. (2003). Pricing options on scalar diffusions: an eigenfunction expansion approach. Operat. Res., 51, 185-209. Retrieved from http:// citeseerx.ist.psu.edu/viewdoc/dow nload? $\mathrm{doi}=10.1 \cdot 1 \cdot 525.4805 \& \mathrm{rep}=\mathrm{r}$ ep1\&type $=$ pdf

7. Going-Jaeschke, A., \& Yor, M. (2003). A Survey and Some Generalizations of Bessel Processes. Bernoulli, 9, 313-349.

8. Gorovoi, V., \& Linetsky, V. (2004). Black's model of interest rates as options, eigenfunction expansions and japanese interest rates. Mathematical finance, 14(1), 49-78.
Retrieved from http://citeseerx.ist. psu.edu/viewdoc/download;jsessio nid $=4$ FCA82ABD9FDB1CE7CB0 AAC71C78FD5E?doi=10.1.1.575. $6876 \&$ rep $=$ rep $1 \&$ type $=$ pdf

9. Hull, J., \& White, A. (1987). The pricing of options on assets with stochastic volatilities. The Journal of Finance, 42(2), 281-300. Retrieved from http://faculty.baruch. cuny.edu/lwu/890/hullwhite87.pdf

10. Lebedev, N. N. (1972). Special Functions and Their Applications. New York: Dover.

11. Linetsky, V. (2004). The Spectral Representation of Bessel Processes with Drift: Applications in Queueing and Finance. Journal of Applied Probability, 41, 327-344.

12. Linetsky, V. (2007). Chapter 6 spectral methods in derivatives pricing. In J. R. Birge, V. Linetsky 
(Eds.), Financial Engineering, 15, of Handbooks in Operations Research and Management Science (pp. 223-299). Elsevier. Retrieved from http://users.iems.northwestern.edu/ linetsky/besseldrift.pdf

13. Lorig, M. J. (2014). Pricing Derivatives on Multiscale Diffusions: an Eigenfunction Expansion Approach. Mathematical Finance, 24(2), 331-363.
14. Mendoza-Arriaga, R., Carr, P., \& Linetsky, V. (2010). Time-changed Markov processes in unified credit-equity modeling. Mathematical Finance, 20, 527-569.

15. Merton, R. C. (1973). Theory of Rational Option Pricing. Bell Journal of Economics and Management Science, 4, 141-183.

16. Sharpe, W. (1985). Factor Models, CAPMs and APT. Journal of Portfolio Management, 21-25.
17. Vladimirov, V. S. (1981). Equations of Mathematical Physics. Moscow: Science, 512 p.

18. Yor, M. (1984). On squareroot boundaries for Bessel processes, and pole-seeking Brownian motion. In A. Truman, D. Williams (Eds.), Stochastic Analysis and Applications (Lecture Notes Math. 1095) (pp. 100-107). Springer, Berlin. 